\title{
Development of a dynamic process model for the mechanical fluid separation in decanter centrifuges
}

\author{
Marco Gleiß ${ }^{\mathrm{a}}$, Hermann Nirschl ${ }^{\mathrm{a}}$ \\ ${ }^{a}$ Institute for Mechanical Process Engineering and Mechanics, Karlsruhe Institute of \\ Technology (KIT), Karlsruhe 76131, Germany \\ marco.gleiss@kit.edu; www.mvm.kit.edu
}

\begin{abstract}
The flowsheet simulation is a current simulation tool in the areas of research \& development, plant design and production of chemical products. The objective of flowsheet simulations is the estimation of the overall process behaviour by solving mass and energy equations. The dynamic flowsheet simulation of particulate processes is a new tool which extends the classical simulation tools which are used in the chemical process engineering by the particle size distribution as one relevant new parameter. The complexity of particulate processes is given by the variety of material properties which depend on the particle size, particle size distribution, the volume fraction of solids and the physicochemical properties.

This work illustrates a dynamic process model for continuous centrifuges and considers the material properties of the processed solids and the machine behaviour. First the theoretical framework is presented. Due to the complexity of the material, different material functions, such as the particle size distribution, hindered settling, consolidation or sediment transport used for the dynamic calculation of the process within a lab-decanter centrifuge. Example simulations show the capability of the presented approach. One main feature of the numerical approach is given by the fact, that the small numerical effort reveals the possibility for real time simulations which are applied in the process industry for calculation at the same rate as actual wall clock time.
\end{abstract}

\section{KEYWORDS}

Solid-Liquid separation, Decanter Centrifuges, Real Time Simulations, Dynamic Process

Control, Dynamic Process Model 


\section{Introduction}

The dynamic flowsheet simulation as computation tool is used in the area of research \& development, design and production of chemical plants. The main goal of process simulation is the connection between laboratory and pilot scale to the production plant. Thus, the production plant is built by several different unit operations. In recent years, the concept of flowsheet simulation was extended from the chemical process engineering to the simulation of particulate processes. Flowsheet simulation covers several aspects of process engineering, such as oscillations in the process behaviour, design and operation or dynamic control and optimization (Dosta et al., 2010). An overview of different flowsheet simulation tools for particulate processes is shown in (Dosta, 2013). The dynamic flow sheet simulation occupies the calculation of the transient process behaviour by solving of mass and energy balances for every process unit. This enables the calculation of serial circuit of different unit operations or tear stream connections.

One major difficulty for characterization of particulate processes is the polydisperity of the solids phase. In the case of solids-liquid separation the product shows complex material properties. Therefore, the treatment of the suspension results in a variety of different process units (Anlauf, 2007). Due to high operating costs and environmental aspects, the solid-liquid separation is applied by connecting of several solid-liquid separation units, such as thickeners, hydrocyclones, filters or centrifuges. In the case of centrifugation the processed suspension passes different states from a dilute suspension to a dense networked cake. For this purpose, the gel point defines the transition between particle settling and networked particles, whereby stresses can be transferred due to the continuous contact of particles. The dynamic modelling of solid-liquid separation is still a challenging task of research and development which results in several studies for the modelling of the sedimentation and consolidation processes in thickeners which is given in ( $\mathrm{Li}$ and Stenstrom, 2014). The main difference between the thickening process in a secondary clarifier and the separation process in a centrifuge is the flow direction of the liquid. The main liquid flow in a clarifier occurs upward and downward of the process unit which enables a simplified 1D-modelling approach for the separation process. Different behaviour is given for semi-batch or continuous centrifugation processes where the main fluid flow is not in the direction of the separation process. Nevertheless, the modelling of continuous centrifugation processes reveal in a 2D- or 3D-modelling of the process behaviour. (Ambler, 1959, 1961, 1952) applied the process design of centrifuges by considering the settling path of a single particle with the ideal flow behaviour at steady-state conditions in centrifuges.

This paper focusses on the dynamic process modelling of centrifuges by considering material properties of the solid-liquid mixture and the machine behaviour of a centrifuge. By solving the transient mass balance of the solid phase and considering the particle size distribution enables the calculation of the fluid transport along the screw channel and the sediment buildup. The non-ideal flow in the centrifuge is modelled by the residence time distribution which describes the residence probability of particles which not separated. Exemplary calculations for the ramp change of the particle size distribution at the inlet shows the application of the presented approach to simulations of the dynamic process behaviour. 


\section{Theory}

The design of centrifuges is based on the Stokesian settling of a single particle of spherical shape in an infinite dilute regime. The Stokesian settling velocity is derived by balancing the centrifugal force, buoyancy and drag force. The Stokes settling velocity in the centrifugal field

$$
u_{s t}=\frac{\left(\rho_{s}-\rho\right) \cdot d_{p}^{2} \cdot c g}{18 \cdot \eta_{l}}
$$

depends on the densities of the pure solid $\rho_{s}$ and liquid $\rho_{l}$, the viscosity of the liquid $\eta_{l}$, the relative centrifugal number $C$, the gravitational acceleration $g$ and the particle diameter $d_{p}$. Eq. (1) is only valid for a creeping flow with $R e<0.25$. Additionally, the impact of particle interaction on the solids settling by increasing the solids concentration is neglegted. $A$ detailed description of the settling of particles in the centrifugal field is shown in (Leung, 1998). However, the Stokes settling is only valid for the settling of a particle without walls and reveals no information about the settling within a centrifuge. Therefore the sigma theory connects the settling time of a particle derived from Eq. (1) with the mean residence time in a centrifuge. By considering ideal flow behaviour, the mean residence time $t_{\text {res }}$ is given by the ratio of centrifuge volume and liquid throughput:

$$
t_{\text {res }}=\stackrel{\forall}{Q}
$$

where $V$ is the volume of the empty centrifuge and $Q$ is the liquid throughput at the inlet. A detailed description of the centrifuge performance calculation is presented in (Beiser, 2006; Leung, 1998; Stahl, 2004). Due to the flow conditions within the centrifuge. By comparing the settling time of a particle with a specific size, the mean residence time results in the critical settling position of a particle.

$$
R_{\text {sep }}=R_{b} \cdot \exp \left\{\begin{array}{c}
-\frac{\left(\rho_{s}-\rho_{f}\right) \cdot R(\phi) \cdot d_{p}^{2} \cdot \omega_{b}^{2}}{18 \cdot \eta_{f}} \cdot \frac{A \cdot L_{c t f}}{Q} \\
Q
\end{array}\right\}
$$

where $R_{\text {sep }}$ is the critical settling position, $R_{b}$ is the bowl radius of the centrifuge, $A$ is the cross-sectional area of the flow region, $L_{c l f}$ is the length of the cylindrical drum and $Q$ is the feed flow rate. The liquid forms a channel flow along the screw conveyor of the decanter centrifuge. Therefore, the calculation of the flow cross-sectional area divers from the conventional calculation of tubular or solids bowl centrifuges without internals. By neglecting the curvature of the drum, the cross-sectional area of the flow region

$$
A=W_{s c} \cdot\left(R_{b}-R_{w}\right)
$$

is given by the product of the screw pitch $W_{s c}$ and the height of the channel $R_{b}-R_{w}$. The grad efficiency is the separation probability related to the particle size and is a significant parameter for performance calculation of a centrifuge.

$$
T\left(d_{p}\right)=\frac{R_{b}}{R_{b}-R_{w}} \cdot\left(1-\left[\exp \left\{\begin{array}{c}
-\left(\rho_{s}-\rho_{f}\right) \cdot R(\phi) \cdot d_{p}^{2} \cdot \omega_{t}^{2} \\
18 \cdot \eta_{l}
\end{array} \cdot \frac{\left(R_{b}-R_{w}\right) \cdot W_{S c} \cdot L_{c t f}}{Q_{i n}}\right\}\right]\right)
$$


The presented correlation for the grade efficiency curve $T\left(d_{p}\right)$ is valid for the separation process of particles transported along the screw channel of a decanter centrifuge and depends on machine parameters and material properties. The increase of solids concentration leads to a growth of momentum exchange between particles and fluid. Therefore, the settling velocity of the solids phase derives from Stokes settling conditions. However, the increase of momentum exchange is considered by a drag correction by introducing the hindered settling function $R(\phi)$. Table 1 shows an overview of different hindered settling functions. In consequence of the operation conditions which are at high solids throughput the hindered settling functions is of high importance. Nevertheless, for a successful design of centrifuges the batch centrifugation test is an adequate method for characterization of the settling behaviour dependent on the particle size distribution, solids concentration physicochemical properties and the centrifugal acceleration.

Table 1: Overview about different hindered settling models

\begin{tabular}{|c|c|c|}
\hline $\begin{array}{l}\text { (Richardson and } \\
\text { Zaki, 1997) }\end{array}$ & $R(\phi)=\frac{u}{u_{s t}}=(1-\phi)^{n}$ & $\begin{array}{l}\text { - spherical shaped particles } \\
\text { - } n=4.65 \text { for } R e<0.25\end{array}$ \\
\hline $\begin{array}{l}\text { (Ekdawi and } \\
\text { Hunter, 1985) }\end{array}$ & $R(\phi)=\frac{u}{u_{s t}}=(1-\phi)^{2}\left[1-\frac{\phi}{\phi_{\max }}\right]^{2.5 \cdot \phi_{\max }}$ & $\begin{array}{l}\text { - empirical parameter } \phi_{\max } \\
\text { characterized by experiments }\end{array}$ \\
\hline $\begin{array}{l}\text { (Michaels and } \\
\text { Bolger, 1962) }\end{array}$ & $R(\phi)=\frac{u}{u_{s t}}=\left(1-\frac{\phi}{\phi_{\max }}\right)^{n_{m b}}$ & $\begin{array}{l}\text { - considers the influence on solids } \\
\text { packing on hindered settling by } \phi_{\max } \\
\text { - } n_{m b}>1 \text { is an empirical parameter } \\
\text { with }\end{array}$ \\
\hline (Scott K.J., 1968) & $R(\phi)=\frac{u}{u_{s t}}=(1-k \cdot \phi)^{n}$ & $\begin{array}{l}-k \text { and } n \text { are empirical parameter, } \\
\text { which takes the increase of solids } \\
\text { concentration due to aggregates into } \\
\text { account }\end{array}$ \\
\hline
\end{tabular}

\section{Simulation procedure}

Centrifuges are operated at steady-state conditions. Dynamic effects occur at the spin-up process and by changing material properties or process conditions. The sigma approach as one theory for the design of centrifuge and is restricted to steady-state conditions. This fact reveals no statement about the dynamic behaviour of centrifuges. One opportunity to describe the deviation from ideal flow is the estimation of the residence time distribution of the solids within the centrifuge. A deviation from ideal flow occurs due to back-mixing, recirculation or dead-zone, whereby these effects are unrecognized by state of the art models.

Fig. 1 shows the schematic representation of the compartment model for a counter-current decanter centrifuge. The centrifuge is divided into 25 compartments. The numerical approach calculates the mass conservation of the clarification zone and the sediment build-up for every time step by solving Eq. (8) Eq. (9) and Eq. (10). The calculation of the sediment build-up takes the reduction of the cross-sectional area due to the increase of sediment layer under consideration. 


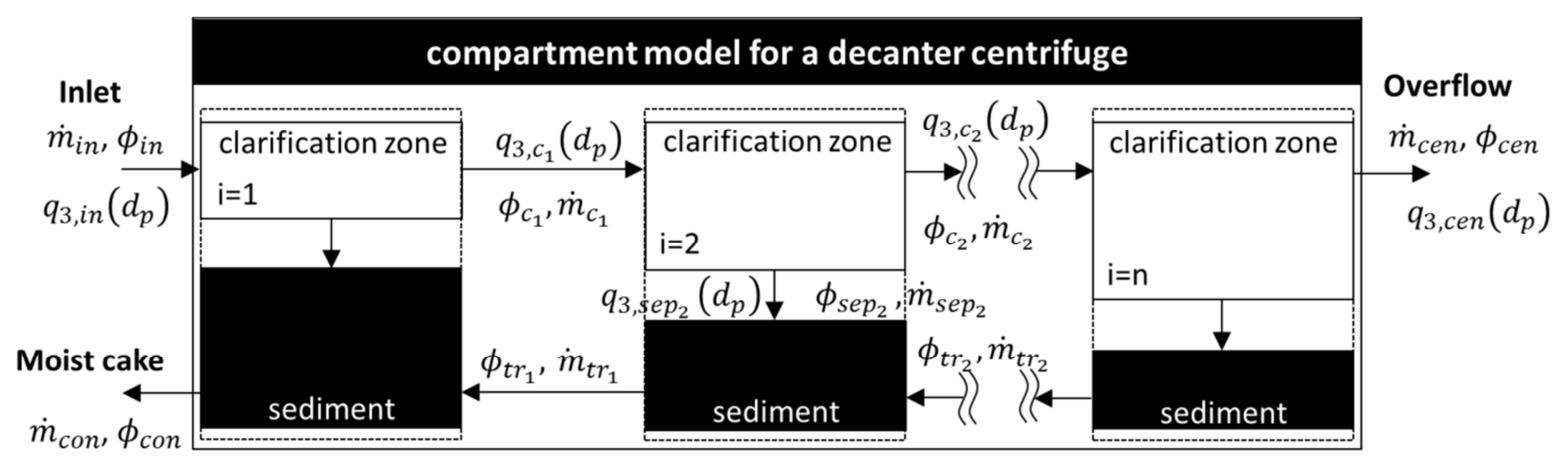

Fig.1: Schematic representation of the compartment approach for the clarification zone of a decanter centrifuge

By dividing the centrifuge intro a finite number of compartments $(n=25)$ reveals in a set of ordinary differential equations which have been solved for every time step $\Delta$ t. The solids mass conservation for the separation process in the clarification zone of compartment 2 is given by:

$$
\dot{m}_{s, c_{1}}=\dot{m}_{s, s e p_{2}}+\dot{m}_{s, R T D_{2}}
$$

where $\dot{m}_{s, c_{1}}$ is the solids mass stream of the feed, $\dot{m}_{s, s e p_{2}}$ is the mass stream of separated solids and $\dot{m}_{S, R T D_{2}}$ is the mass stream which connects the steady-state behaviour of the centrifuge with the residence time distribution. The separation efficiency

$$
\eta_{\text {sep }_{2}}=\int_{d_{p, \min }}^{d_{p, \max }}\left[T_{\text {sep }_{2}}\left(d_{p}\right) \cdot q_{3, c_{2}}\left(d_{p}\right)\right] d d_{p}
$$

represents an important factor for the performance calculation of a centrifuge and is shown by Eq. (7), where $T_{s e p_{2}}\left(d_{p}\right)$ represents the grade efficiency curve and $q_{3, c_{2}}\left(d_{p}\right)$ the density distribution function at the feed. By considering the feed flow rate $Q_{2}$ and the solids volume fraction $\phi_{c_{2}}$ at the inlet of compartment 2 results in the mass stream out of the steady-state separator.

$$
\dot{m}_{s, R T D_{2}}=Q_{2} \phi_{c_{2}} \cdot\left\{1-\int_{d_{p, \min }}^{d_{p, \max }}\left[T_{s e p, 2}\left(d_{p}\right) \cdot q_{3,2}\left(d_{p}\right)\right] d d_{p}\right\}
$$

The residence time distribution characterizes the deviation from ideal flow behaviour. The serial-circuit connection of different compartments takes the non-ideal flow by considering the dispersion in every compartment by a "black-box" system into account. Dispersion in a centrifuge occurs because of turbulent flow conditions, the polydisperity of the particulate system and gradients in solids concentration along the flow channel. The residence time distribution modelling allows a fast simulation of the transient separation process within a decanter centrifuge and can be used for real-time simulations. Thus, the unsteady balance of an ideal back-mixed compartment is given by:

$$
\frac{d\left(m_{\left.s, c_{2}\right)}\right)}{d t}=\dot{m}_{s, R T D_{2}}-\dot{m}_{s, c_{2}}
$$


where $m_{s, c_{2}}$ is the hold-up of compartment $2, \dot{m}_{s, R T D_{2}}$ is the mass stream inside the ideal back-mixed compartment and $\dot{m}_{s, c 2}$ is the mass stream into compartment 3 . The calculation of the dynamic transport balance for the moist cake, enables the calculation of the sediment layer height and the reduction of the cross-sectional area of the clarification zone.

$$
\frac{d m_{s, t r_{2}}}{d t}=\dot{m}_{s, s e p_{2}}+\dot{m}_{s, t r_{2}}-\dot{m}_{s, t r_{1}}
$$

$m_{s, t r_{2}}$ is the accumulation of solids, $\dot{m}_{s, t r_{2}}$ is the transport mass stream into compartment 2 and $\dot{m}_{s, t r_{1}}$ is the transport mass stream out of compartment 2 . The mass stream of the separated solids from the clarification zone is calculated by Eq. (6). The material properties of the networked particles influence the transport behaviour of the screw conveyor. Usually, flocculated systems build pasty materials with time-dependent consolidation behaviour, whereby stabilized system generate approximately incompressible cakes. For a single incompressible body with uniform shape, the mass stream of transported solids along the drum wall is given by:

$$
\dot{m}_{s, t r_{2}}=(1-\varepsilon) \rho_{s} W_{s c} \cdot \Delta H_{2} \cdot u_{t r_{2}}=\phi_{s e d} \rho_{s} W_{s c} \cdot\left(R_{d}-R_{t r_{2}}\right) \cdot u_{t r_{2}}
$$

where $\varepsilon$ is the porosity of the cake, $\Delta H_{2}$ is the height of the accumulated solids and $R_{t r_{2}}$ is the radial position of the upper sediment layer. For the transport of the moist cake we assume a uniform transport velocity:

$$
u_{t r_{i}}=\frac{W_{S c} \Delta \omega \cdot E}{\sin (\beta)}=\frac{W_{s c} \Delta \omega}{(1+\tan (\beta) \tan (\kappa)) \cdot \sin (\beta)}
$$

where $\Delta \omega$ is the differential speed between drum and screw conveyor, $E$ is the transport the transport efficiency, $\beta$ is the screw angle and $\kappa$ transport angle. The transport efficiency describes the deviation from purely axial movement and is derived from a trigonometric function of the screw and transport angle. A detailed description of incompressible cake transport in decanter centrifuges is shown by (F.A. Records, 1974; Reif and Stahl, 1989; Stahl, 2004; Stickland, 2005).

\section{Results \& Discussion}

\begin{tabular}{|rr|}
\hline \multicolumn{2}{|c|}{ Table 2: Overview about initial conditions for the numerical simulation } \\
\hline Weir radius $\left(R_{w}\right)$ & $0.034 \mathrm{~m}$ \\
\hline Drum radius $\left(R_{d}\right)$ & $0.04 \mathrm{~m}$ \\
\hline Screw pitch $\left(W_{s c}\right)$ & $0.025 \mathrm{~m}$ \\
\hline Width of a screw blade $\left(W_{s b}\right)$ & $0.002 \mathrm{~m}$ \\
\hline Length of the cylindrical drum $(L)$ & $0.176 \mathrm{~m}$ \\
\hline Length of the centrifuge $\left(L_{c}\right)$ & $0.21 \mathrm{~m}$ \\
\hline Cone angle $(\alpha)$ & $7^{\circ}$ \\
\hline Centrifugal acceleration $(C g)$ & 11 to $4000 \cdot \mathrm{g}$ \\
\hline Centrifuge volume $\left(V_{c}\right)$ & $230 \mathrm{~mL}$ \\
\hline Number of segments $(n)$ & 25 \\
\hline Number of particle classes $(j)$ & 100 \\
\hline
\end{tabular}


FILTECH 2016 - L4 - Decanter and Tube Centrifuges

\begin{tabular}{|rr|}
\hline Time step size $(\Delta t)$ & $1 \mathrm{~s}$ \\
\hline Density of polyvinylchloride $\left(\rho_{s}\right)$ & $1410 \mathrm{~kg} \cdot \mathrm{m}^{-3}$ \\
\hline Density of water $\left(\rho_{l}\right)$ & $998 \mathrm{~kg} \cdot \mathrm{m}^{-3}$ \\
\hline Dynamic viscosity of water $\left(\eta_{l}\right)$ & $0.001 \mathrm{~Pa} \cdot \mathrm{s}$ \\
\hline Friction coefficient PVC $\left(\mu_{s}\right)$ & 0.3 \\
\hline Differential speed $(\Delta n)$ & $5 \mathrm{rpm}$ \\
\hline Hindered settling function & $R(\phi)=\left(1-\frac{\phi}{\phi_{\max }}\right)^{4.65}$ \\
\hline Maximum solids volume fraction $\left(\phi_{\text {sed }}\right)$ & 0.55 \\
\hline Particle size distribution $(\mathrm{PSD})$ & $Q_{3}\left(d_{p}\right)=a_{2}+\frac{a_{1}+a_{2}}{\left(1+\frac{d_{p}}{d_{p, 50}}\right)^{b}}$ \\
\hline & \\
\hline Fit parameter PSD & $a_{1}=0, a_{1}=1, b=4.07, d_{p, 50}=2.29 \cdot 10^{-6} \mathrm{~m}$ \\
\hline
\end{tabular}

Table 2 shows an overview about the initial conditions for the centrifuge geometry and material properties. The change of material properties, such as the particle size distribution or the solids volume fraction has a significant impact on the separation performance of a centrifuge, because the grade efficiency curve of a centrifuge is influenced by the particle size and the solids volume fraction.

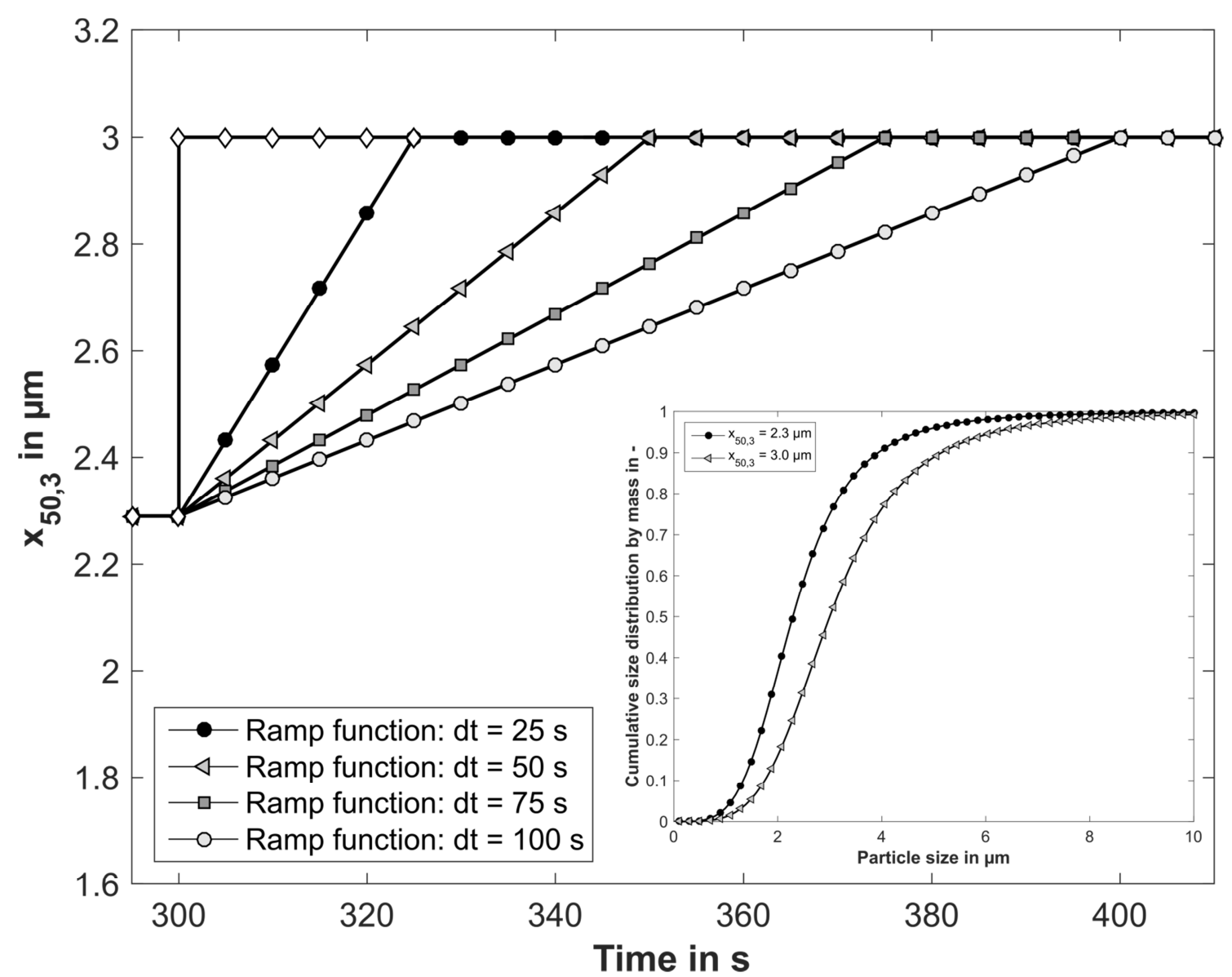

Fig. 2: Temporal change of the mean particle size at the inlet; Small picture: Initial and final states for changing the particle size distribution 
A change of the particle size to smaller values show negative effects for the separation performance of centrifuges. However, the change of material properties for particles in the micron range modifies the product quantities and reveal in a different separation behaviour and sediment build-up. In the worst case, this lead to a totally break down of the process.

At this point, the dynamic flowsheet simulation is an appropriate tool for characterization of product changes. The fast simulation tool allows real-time simulations of the process behaviour. Real-time simulations allocate a physical based approach and executed at the same rate as the actual wall clock time. The dynamic approach is presented for example simulations for the product polyvinylchloride for a lab-decanter centrifuge MD80 from Lemitec $\mathrm{GmbH}$. The top of Fig. 2 shows the mean particle size dependent on time. The small illustration shows the initial and final state of the particle size distribution. The given ramp function shifts the particle size distribution at the inlet after dependent on time until the new steady-state is reached.

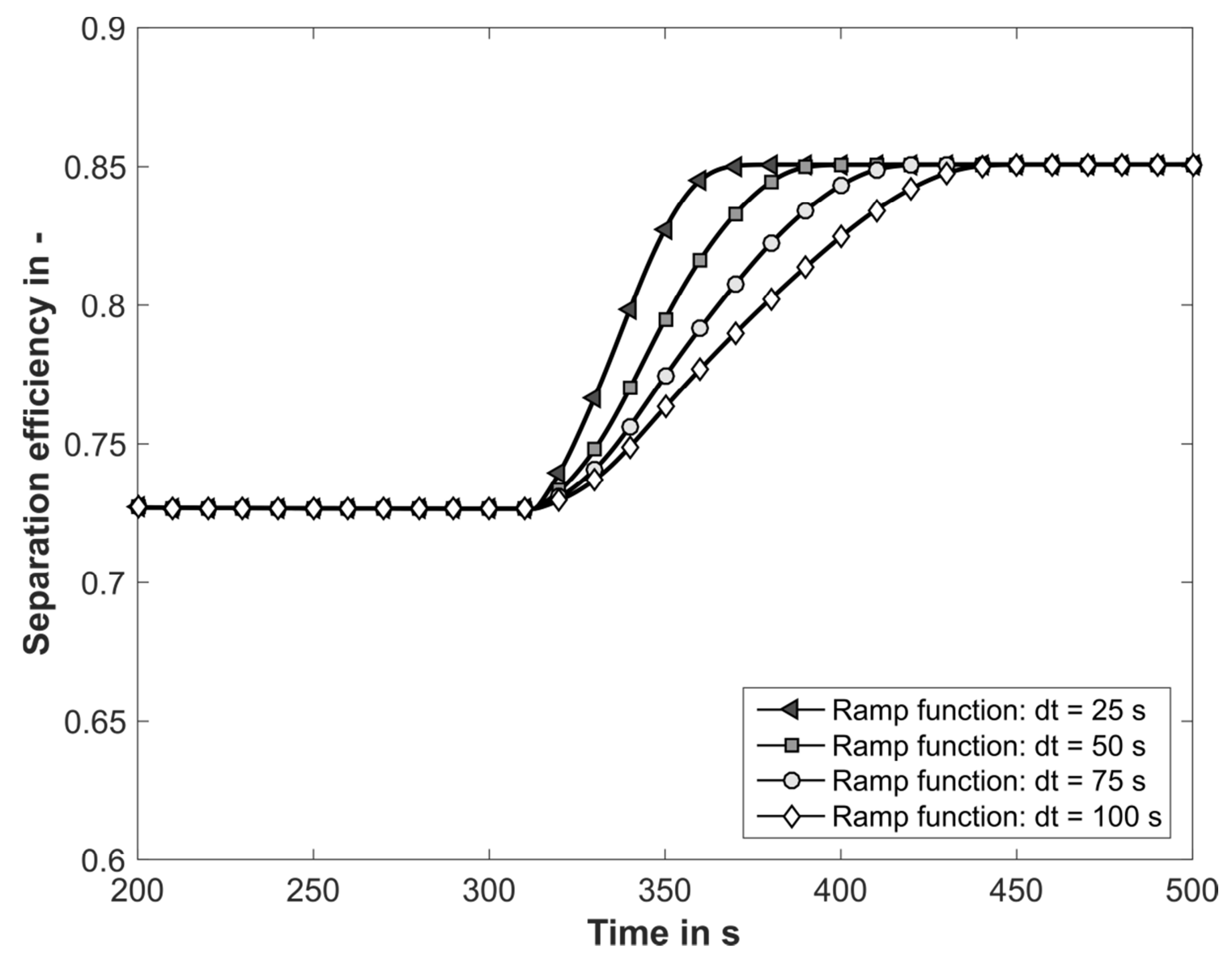

Fig. 3: Separation efficiency against time for the temporal shift of particle size distribution to higher particle sizes for $Q=30 \mathrm{Lh}^{-1}$ and $\mathrm{C}=250 \mathrm{~g}$ and a solid volume fraction of $\phi_{\text {in }}=0.02$

Fig.3 exemplifies the change of the separation efficiency dependent on time for the previously presented ramp change of the particle size distribution at the inlet of the centrifuge. The simulations are performed for a flow rate of $Q=30 \mathrm{Lh}^{-1}$ a centrifugal acceleration of $C=250 \mathrm{~g}$ and a solids volume fraction at the inlet of $\phi_{\text {in }}=0.02$. The change of particle size distribution results in a temporal change of solids concentration at the overflow. This fact is illustrated by the change of separation efficiency after a time of $t=$ $300 \mathrm{~s}$. The overall time until the centrifuge reaches the new steady-state depends on the 
geometry and the flow behaviour of the centrifuge. Both parameters affect the residence time distribution of the centrifuge and the dynamic behaviour. The presented results illustrate the capability of the presented numerical approach for dynamic flowsheet simulations and process optimization and control. Moreover, compressible materials can be investigated by the presented approach by considering the material properties and consolidation behaviour of those kind of slurries.

\section{Conclusions}

A compartment model for the clarification process in a counter-current decanter centrifuge was presented. Based on the flowsheet simulation concept, the mass balance was modelled by connecting the material properties and the residence time distribution. The modelling approach is designed for the calculation of temporal changes of the separation efficiency by changing material properties or process conditions. The approach supports a unified framework for the simulation of separation efficiency and sediment build-up of incompressible materials under both steady-state and dynamic terms. Moreover, the presented approach is applicable for tubular or solid bowl centrifuges by considering the geometry of and residence time distribution of these machines.

\section{Acknowledgements}

The authors would like to thank the German Research Foundation (DFG) for funding this project under the Priority Program SPP 1679 "Dynamic simulation of interconnected solids processes".

\section{References}

Ambler, C., 1959. The theory of scaling up laboratory data for the sedimentation type centrifuge. J Biochem Microbiol Technol Eng 1, 185-205.

Ambler, C.M.., 1952. The evaluation of centrifuge performance. Chem. Engneering Prog. 48, 150-158.

Ambler, C.M.., 1961. The fundamentals of separation, including SHARPLES Sigma value for predicting equipment performance. Ind. Eng. Chem. 48, 430-433.

Anlauf, H., 2007. Recent developments in centrifuge technology. Sep. Purif. Technol. 58, 242-246.

Beiser, M., 2006. Sedimentationsverhalten submikroner Parikeln in Abhängigkeit physikalisch-chemischer Einflüsse und ihr Separationsverhalten in Dekantierzentrifugen. Universität Karlsruhe (TH), Dissertation.

Dosta, M., 2013. Dynamic Flowsheet Simulation of Solids Processes and its Application to Fluidized Bed Spray Granulation. Technical University Hamburg-Harburg.

Dosta, M., Heinrich, S., Werther, J., 2010. Fluidized bed spray granulation: Analysis of the system behaviour by means of dynamic flowsheet simulation. Powder Technol. 204, 7182.

Ekdawi, N., Hunter, R.J., 1985. Sedimentation of disperse and coagulated suspensions at high particle concentrations. Colloids and Surfaces 15, 147-159.

F.A. Records, 1974. The continous scroll dischage decanting centrifuge. Chem. Eng. 281.

Leung, W.W.-F., 1998. Industrial Centrifugation Technology. McGraw-Hill, New York. 
Li, B., Stenstrom, M.K., 2014. Research advances and challenges in one-dimensional modeling of secondary settling Tanks - A critical review. Water Res. 65, 40-63. doi:10.1016/j.watres.2014.07.007

Michaels, A.S., Bolger, J.C., 1962. Settling Rates and Sediment Volumes of Flocculated Kaolin Suspensions. Ind. Eng. Chem. Fundam. 1, 24-33.

Reif, F.R., Stahl, W., 1989. Transportation of moist solids in Decanter Centrifuges. Chem. Engneering Prog. 85, 57-67.

Richardson, J.F., Zaki, W.N., 1997. Sedimentation and fluidisation: Part I. Chem. Eng. Res. Des. 75, 82-100.

Scott K.J., 1968. Experimental Study of Continuous Thickening of a Flocculated Silica Slurry. Ind. Eng. Chem. Fundam. 7, 582-595.

Stahl, W., 2004. Fest-Flüssig-Trennung Band II: Industrie-Maschinen- und Verfahrenstechnik. DRM Press, CH-Männedorf.

Stickland, A.D., 2005. Solid Liquid Separation in Water and Wastewater Industries. University of Melbourne, Department of Chemical and Biomolecular Engineering. 